El ect rost at i c i nt er act i on ef fects on tensi on- $\mathrm{i}$ nduced por e for mat $\mathrm{i}$ on i $\mathrm{n} \mathrm{I}$ i pi $\mathrm{d}$ nembr anes

\begin{tabular}{|l|l|}
\hline 著者 & $\begin{array}{l}\text { Kar al Nohammad Abu Sayem Levadnyy Vi ct or, } \\
\text { Tsuboi Taka aki, Bel aya Mar i na, Yamazaki } \\
\text { Nasahi to }\end{array}$ \\
\hline $\begin{array}{l}\text { j our nal or } \\
\text { publ i cat i on ti t l e }\end{array}$ & Physi cal Revi ew E \\
\hline vol une & 92 \\
\hline nunber & 1 \\
\hline page r ange & 012708 \\
\hline year & $2015-07-06$ \\
\hline 出版者 & Amer i can Physi cal Soci et y \\
\hline 権利 & ( C) 2015 Amer i can Physi cal Soci et y \\
\hline URL & ht t p: //hdl . handl e. net /10297/8959 \\
\hline
\end{tabular}




\section{Electrostatic Interaction Effects on Tension-Induced Pore Formation in Lipid Membranes}

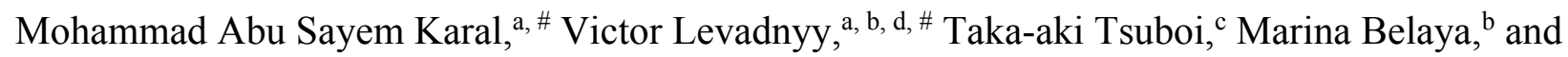
Masahito Yamazaki a, c, d, *

${ }^{a}$ Integrated Bioscience Section, Graduate School of Science and Technology, Shizuoka University,

Shizuoka 422-8529, Japan

${ }^{b}$ Theoretical Problem Center of Physico-Chemical Pharmacology, Russian Academy of Sciences, Kosugina,

4, 117977, Moscow, Russia

${ }^{c}$ Department of Physics, Graduate School of Science, Shizuoka University, Shizuoka 422-8529, Japan

${ }^{d}$ Nanomaterials Research Division, Research Institute of Electronics, Shizuoka University, Shizuoka 422-8529, Japan

PACS: 87.16.dm, 87.16.dj, 41.20.Cv, 05.10.Gg.

\# These authors contributed equally to the study.

*Correspondence should be addressed to: Dr. Masahito Yamazaki

Nanomaterials Research Division, Research Institute of Electronics,

Shizuoka University

836 Oya, Suruga-ku, Shizuoka 422-8529, Japan

Tel/Fax: 81-54-238-4741; E-mail: yamazaki.masahito@shizuoka.ac.jp 


\begin{abstract}
We investigated the effects of electrostatic interactions on the rate constant $\left(k_{\mathrm{p}}\right)$ for tension-induced pore formation in lipid membranes of giant unilamellar vesicles under constant applied tension. A decrease in salt concentration in solution as well as an increase in surface charge density of the membranes increased $k_{\mathrm{p}}$. These data indicate that $k_{\mathrm{p}}$ increases as the extent of electrostatic interaction increases. We developed a theory on the effect of the electrostatic interactions on the free energy profile of the membrane containing a prepore and also on the values of $k_{\mathrm{p}}$, which explains the experimental results and fits the experimental data reasonably well in the presence of weak electrostatic interactions. Based on these results, we conclude that a decrease in the free energy barrier of the prepore state due to electrostatic interactions is the main factor causing an increase in $k_{\mathrm{p}}$.
\end{abstract}




\section{Introduction}

Lateral tension is induced in biomembranes or lipid membranes if external forces are applied to cells or liposomes. In most cases this tension is positive, which induces stretching of the membranes. When a large positive tension is applied on a biomembrane, pore formation occurs in the membrane, causing rupture of cells or liposomes. To reveal the kinetics and the mechanism of tension-induced pore formation, giant unilamellar vesicles (GUVs) have been used [1-5]. By the use of a viscous solvent, Brochard-Wyart et al. visualized directly at video rate the fast dynamics of pore openings and closings in a GUV which was stretched as a result of intense optical illumination [1]. Evans et al. investigated the rupture of a GUV under dynamic applied tension, in which ramps of tension with loading rates (i.e., tension/time) were applied using a micropipet $[2,3]$. Recently we developed a theory to determine the rate constant $k_{\mathrm{p}}$ of constant tension-induced pore formation in lipid membranes as a function of tension $\sigma$ using the mean first passage time (MFPT) concept [6].

In biomembranes such as cell membranes, there are many negatively charged lipids. Electrostatic interactions due to these charged lipids play important physiological roles such as binding of proteins to cell membranes, structural changes of membranes, and membrane stability [7-9]. However, the effect of these charged lipids on the mechanical stability of membranes is not well understood. In this report, we systematically consider the effects of electrostatic interactions on constant tension-induced pore formation in GUVs. Using the method developed in our previous paper [6], we measured the rate constant $k_{\mathrm{p}}$ of constant tension-induced pore formation in GUVs composed of mixtures of negatively charged dioleoylphosphatidylglycerol (DOPG) and neutral dioleoylphosphatidylcholine (DOPC). We examined the effects of salt concentration, $C$, and of DOPG molar fraction in the lipid membrane, $X$, on the $k_{\mathrm{p}}$ values. Based on these results, we discuss the possible mechanism behind the effect of electrostatic interactions on tension-induced pore formation.

\section{Materials and Methods}

DOPG and DOPC were purchased from Avanti Polar Lipids Inc. (Alabaster, AL). Bovine serum albumin (BSA) was purchased from Wako Pure Chemical Industries Ltd. (Osaka, Japan). For experiments on salt concentration effects, $40 \%$ DOPG $/ 60 \%$ DOPC GUVs (where \% indicates mole \%) were prepared in 
buffer A (10 mM PIPES, pH 7.0, $1 \mathrm{mM}$ EGTA) containing various concentrations of $\mathrm{NaCl}$ and $0.1 \mathrm{M}$ sucrose by natural swelling [6]. For experiments on surface charge density effects, GUVs of DOPG/DOPC membranes containing various molar fractions of DOPG were prepared in buffer A containing $150 \mathrm{mM} \mathrm{NaCl}$ and $0.1 \mathrm{M}$ sucrose. To purify these GUV suspensions, the membrane filtering method was used [10]. The purified GUV suspensions $(300 \mu \mathrm{L})(0.1 \mathrm{M}$ sucrose in buffer as the internal solution; $0.1 \mathrm{M}$ glucose in buffer as the external solution) were transferred into a hand-made microchamber. The GUVs were observed by fluorescence and differential interference contrast imaging using an inverted microscope (IX-71, Olympus, Tokyo, Japan) at $25 \pm 1{ }^{\circ} \mathrm{C}$ using a Thermoplate microscope stage thermocontrol system (Tokai Hit, Shizuoka, Japan). These images were recorded using a CS230B CCD camera (Olympus) with a DVD recorder.

To obtain $k_{\mathrm{p}}$, we used the method [6] as follows. To apply tension to lipid membranes of single GUVs, we initially held the GUV at the tip of a micropipet for a few minutes using only slight aspiration pressure (i.e., the difference in pressure between the outside and the inside of a micropipet, $\Delta P$ ) (Fig. 1a), with a tension on the bilayer of $\sim 0.5 \mathrm{mN} / \mathrm{m}$, and then rapidly $(\sim 10 \mathrm{~s})$ increased $\Delta P$ to reach a specific level of tension, and held this tension until the GUV was completely aspirated into the inside of the micropipet. The time of pore formation was defined as the time when the GUV was completely aspirated, and has a time resolution of less than $1 \mathrm{~s}$. The tension of the GUV membrane, $\sigma$, can be described as a function of $\Delta P$ as follows [11]:

$$
\sigma=\frac{\Delta P d}{4(1-d / D)},
$$

where $d$ is the internal diameter of the micropipet and $D$ is the diameter of the spherical part of the GUV exterior to the micropipet (Fig. 1a). Micropipets were coated with $0.5 \%(\mathrm{w} / \mathrm{v})$ BSA in buffer containing 0.1 $\mathrm{M}$ glucose and glass surfaces in chambers were coated with $0.1 \%(\mathrm{w} / \mathrm{v}) \mathrm{BSA}$ in the same buffer.

\section{Experimental Results}

We investigated the effects of salt concentration on tension-induced pore formation in GUVs containing $40 \%$ DOPG and $60 \%$ DOPC (i.e., $X=0.40$ ). The first experiment used buffer A (10 mM PIPES, $\mathrm{pH} 7.0,1$ 
mM EGTA) without $\mathrm{NaCl}$ (total salt concentration $C$ was $12 \mathrm{mM}[12]$ and Debye length, $\lambda_{\mathrm{D}}=1 / \kappa$, was $2.8 \mathrm{~nm}$ ). We applied constant tension to a GUV using a micropipet and then observed the GUV until the GUV was suddenly aspirated into the micropipet due to pore formation in the GUV membrane [6]. Then we performed the same experiments with 20 single GUVs (i.e., $n=20$ ), and found that pore formation occurred stochastically at different times. To determine $k_{\mathrm{p}}$ experimentally, we analyzed this stochastic phenomenon statistically [6]. Figure $1 \mathrm{~b}$ shows the time course of the fraction of intact GUVs out of all examined GUVs, $P_{\text {intact }}(t)$, at $\sigma=5.0 \mathrm{mN} / \mathrm{m}$ or $\sigma=3.0 \mathrm{mN} / \mathrm{m}$. The time courses for $P_{\text {intact }}(t)$ were good fits to a single exponential decay function as follows.

$$
P_{\text {intact }}(t)=\exp \left(-k_{\mathrm{p}} t\right)
$$

where $k_{\mathrm{p}}$ is the rate constant for pore formation and $t$ is the duration of constant tension applied to a GUV (tension was started at $t=0$ ). From this fit, we obtained the $k_{\mathrm{p}}$ values, which increased with tension, and were $k_{\mathrm{p}}=1.9 \times 10^{-3} \mathrm{~s}^{-1}$ for $3.0 \mathrm{mN} / \mathrm{m}$ and $k_{\mathrm{p}}=1.1 \times 10^{-2} \mathrm{~s}^{-1}$ for $5.0 \mathrm{mN} / \mathrm{m}$ (Fig. 2a, ○).

Next we investigated pore formation in buffer A containing $300 \mathrm{mM} \mathrm{NaCl}(C=312 \mathrm{mM}, 1 / \kappa=0.54$ $\mathrm{nm}$ ) and obtained $k_{\mathrm{p}}$ values for various values of applied tension (Fig. 2a, $\square$ ). For comparison, the experimental data for $k_{\mathrm{p}}$ of the same GUVs in buffer A containing $150 \mathrm{mM} \mathrm{NaCl}(C=162 \mathrm{mM}, 1 / \kappa=0.76$ nm) [6] are also shown. Figure 2a shows that with a decrease in $C$, the tension required to induce pores decreased and $k_{\mathrm{p}}$ increased at the same tension. Electrostatic interactions in buffer increase with a decrease in salt concentration because shielding of the membrane surface charge by counterions decreases (i.e., the Debye length increases) [7, 13], so Fig. 2a indicates that $k_{\mathrm{p}}$ increases with an increase in the extent of electrostatic interaction.

We also investigated the effects of surface charge density, $\Omega$, which is controlled by $X$, on pore formation. Figure $2 \mathrm{~b}$ shows that with an increase in $X$, the tension required to induce pores decreased. Comparing the data for the same tension, but for different $X, k_{\mathrm{p}}$ increases with an increase in $X$. Therefore, both the results in Fig. 2a and $2 \mathrm{~b}$ clearly show that $k_{\mathrm{p}}$ increases with an increase in the extent of electrostatic interaction due to membrane surface charge.

\section{Theory}


Lipid bilayers are ensembles of lipid molecules, and thereby local thermal fluctuations in the lateral density of the bilayers always exist. The local region of a bilayer where the lateral density of lipid molecules is lower than average is defined as a local density rarefaction, or as a prepore in a bilayer [6]. The extent of a prepore formation increases as the average two-dimensional density decreases. Consequently, the probability and size of a prepore in a strained bilayer increases with tension due to the reconstruction of the system energy profile. Although the structure of prepores has not yet been determined experimentally, recent simulations of the molecular dynamics indicate that a pore or a prepore has a toroidal structure, in which the outer and inner monolayers bend and merge in a pore with an inner wall composed of lipid head groups (Fig. 3a) $[14,15]$. For a pore, this toroidal structure is generally accepted $[16,17]$. Here we assumed that a prepore has the same structure of a pore. Once a prepore with a radius $r$ is formed in the membrane (Fig. 3a), the total free energy of the system changes by an additional free energy (called the free energy of a prepore $U_{0}(r, \sigma)$ ) that consists of two terms: one due to the lateral tension $\sigma$, favoring the expansion of the prepore, and the other due to the line tension of the pore edge $\Gamma$ (the line energy per unit length of a prepore in a lipid membrane), favoring prepore closure; $U_{0}(r, \sigma)=2 \pi \Gamma r-\pi \sigma r^{2}[6,18,19]$. If the radius of a prepore is less than the critical radius, $r_{\mathrm{b}}(=\Gamma / \sigma)$, it closes quickly. However, if the radius reaches $r_{\mathrm{b}}$, the prepore transforms into a pore. The energy barrier, $U_{\mathrm{b}}=U\left(r_{\mathrm{b}}\right)\left(=\pi \Gamma^{2} / \sigma\right)$, is defined as the minimum energy required to create a pore in the membrane from the prepore state. The difference between a prepore and a pore in this report is that a prepore is an instable, small hole in the membrane whose radius is smaller than the critical value and thereby it has a short lifetime whereas a pore is a stable, large hole in the membrane whose radius is larger than the critical value and thereby it cannot be closed. In the experiments of tension-induced pore formation using the micropipet method described in the above section, we can detect only the pore formation, but cannot detect the formation of prepores.

For charged membranes, the effects of electrostatic interactions on tension-induced pore formation must be considered. When a prepore has a toroidal structure, the surface of its wall is charged due to the charged lipid head groups. Therefore, the total free energy of a prepore $U(r, \sigma)$ has to include the proper electrostatic term, $U_{\mathrm{el}}$, 


$$
U(r, \sigma)=U_{0}(r, \sigma)+U_{\mathrm{el}}(r, X, \kappa)=2 \pi \Gamma r-\pi \sigma r^{2}+U_{\mathrm{el}}(r, X, \kappa)
$$

The existence of a double electric layer (DEL) near the membrane surface is the main difference between charged and neutral GUVs. The appearance of a prepore in a charged GUV has two consequences $[17,20]$. One is a reduction or disappearance of the DEL above the prepore, which decreases the total free energy of the system. The other is the appearance of the DEL inside the prepore, which is filled with solution. This increases the total free energy of system. Generally the electrostatic Gibbs free energy density (per unit of area) of the DEL, $g$, can be determined as follows [21, 22]:

$$
g=\int_{0}^{\Omega} \psi_{0}(\widetilde{\Omega}) d \widetilde{\Omega}=\frac{1}{2} \Omega \psi_{\text {sur }},
$$

where $\quad \psi_{0}(\widetilde{\Omega})$ is the surface potential of a membrane at a certain time of charging, $\Psi_{\text {sur }}$ is the surface potential at equilibrium, and $\Omega$ is the surface charge density of the membrane. Hence, to obtain the free energy of the system under consideration, $\Omega$ and $\Psi_{\text {sur }}$ must be determined. $\Omega$ of DOPG/DOPC membranes equals $-e X / A_{0}$, where $e$ is the elementary charge and $A_{0}$ is the cross-sectional area of a lipid molecule in the bilayer under no tension [23] (here we assume that $A_{0}$ of a DOPG molecule is the same as $A_{0}$ of DOPC (= $\left.72.5 \AA^{2}[24]\right)$. For simplicity, we also assume that the structure of the prepore is a cylinder with radius $r$ and height $h(=$ bilayer thickness $=4.0 \mathrm{~nm})$ and the surface (i.e., the wall) of the cylinder is composed of head groups of lipids (DOPG and DOPC) (Fig. 3b). For generalization, we assume that the surface charge density on the prepore wall, $\Omega_{\mathrm{p}}$, is less than that of the GUV surface and $\Omega_{\mathrm{p}}=a \Omega$ (where $a$ is an adjustable parameter $(0 \leq a \leq 1)$, and that $\Omega_{\text {p }}$ (i.e., $a$ ) does not depend on the prepore radius (see the Discussion at the final paragraph in this section 4).

To determine the electric potential distribution $\Psi$, we used the Poisson-Boltzmann equation $\nabla^{2} \Psi=\kappa^{2}(k T / e) \sinh (e \Psi / k T)$, where $k T$ is thermal energy, $\lambda_{D}=1 / \kappa=\sqrt{\varepsilon_{0} \varepsilon_{w} k T / 2 n e^{2}}$ is Debye length of the solution, $\varepsilon_{\mathrm{W}}$ is the relative dielectric constant of water, $\varepsilon_{0}$ is the permittivity of free space, and $n$ is the number density of ions in the bulk (see also Appendix) [13, 21, 22]. To estimate the electrostatic interactions outside the bilayer, we used Lekkerkerker's approach [25]; the free energy density of the DEL near any planar membrane, $g_{\text {sol }}$, is expressed as follows: 


$$
g_{\text {sol }}=2 \Omega\left[\frac{1-q}{p}+\ln (p+q)\right] \frac{k T}{e}
$$

where $p=2 \pi \lambda_{B} X / \kappa A$ and $q=\sqrt{1+p^{2}}, \quad \lambda_{B}$ is the Bjerrum length in water, $\lambda_{B}=e^{2} / 4 \pi k T \varepsilon_{0} \varepsilon_{w}=0.716 \mathrm{~nm}$ at $25^{\circ} \mathrm{C}$. This equation is valid for any values of the surface potential of the membrane. However, for electrostatic interactions inside the prepore, this Lekkerkerker's approach cannot be used, because it can be applied only for $\kappa r>>1$ [25]. This is why we used a linearized Poisson-Boltzmann equation, i.e., $\nabla^{2} \Psi=\kappa^{2} \Psi$, which is valid for $|\Psi|<k T / e=25 \mathrm{mV}$. For cylindrical symmetry (i.e., for the prepore interior), the solution of the equation is (denoting the distance of an arbitrary point from the pore's center as $\rho)$ :

$$
\psi=\psi(\rho)=\frac{\Omega_{\mathrm{p}}}{\kappa \varepsilon_{w} \varepsilon_{0}} \frac{I_{0}(\kappa \rho)}{I_{1}(\kappa r)},
$$

where $I_{0}$ and $I_{1}$ are modified Bessel functions. Here we assumed that the $\varepsilon_{\mathrm{w}}$ value in a narrow prepore is the same as that in bulk water $\left(=78.3\right.$ at $\left.25{ }^{\circ} \mathrm{C}\right)$. Thereby the free energy density of the DEL inside of the prepore, $g_{\text {pore }}$, is given by [26]:

$$
g_{\text {pore }}=\frac{1}{2}[\psi(r)-\psi(0)] \Omega_{\mathrm{p}}=\frac{1}{2} \frac{(a \Omega)^{2}}{\kappa \varepsilon_{w} \varepsilon_{0}} \frac{\left[I_{0}(\kappa r)-I_{0}(0)\right]}{I_{1}(\kappa r)} .
$$

The total electrostatic free energy of the DEL inside a prepore is $2 \pi r h g_{\text {pore }}$, and the total electrostatic free energy above and below the location of a prepore of GUV is $2 \pi r^{2} g_{\text {sol }}$. Thereby, the extra free energy of a prepore due to the electrostatic interaction, $U_{\mathrm{el}}(r)$, is:

$$
\begin{aligned}
& U_{e l}=2 \pi r h g_{\text {pore }}-2 \pi r^{2} g_{\text {sol }}= \\
& -\pi r^{2}\left\{4 \Omega\left[\frac{1-q}{p}+\ln (p+q)\right] \frac{k T}{e}-\frac{\Omega^{2}}{\kappa \varepsilon_{w} \varepsilon_{0}}\left[a^{2}\left(\frac{h}{r}\right) \frac{\left[I_{0}(\kappa r)-I_{0}(0)\right]}{I_{1}(\kappa r)}\right]\right\}
\end{aligned}
$$

and the total free energy of a prepore in a charged GUV is (see eq. (3)):

$$
U(r, \sigma)=2 \pi \Gamma r-\pi r^{2}(\sigma+B)
$$

where 


$$
\begin{aligned}
& B=B(r, \kappa, X)=\left\{4 \Omega\left[\frac{1-q}{p}+\ln (p+q)\right] \frac{k T}{e}-\frac{\Omega^{2}}{\kappa \varepsilon_{w} \varepsilon_{0}}\left[a^{2}\left(\frac{h}{r}\right) \frac{\left[I_{0}(\kappa r)-I_{0}(0)\right]}{I_{1}(\kappa r)}\right]\right\} \approx \\
& \left\{4 \Omega\left[\frac{1-q}{p}+\ln (p+q)\right] \frac{k T}{e}-\frac{\Omega^{2}}{\varepsilon_{w} \varepsilon_{0}} a^{2} \frac{h}{2}\right\}
\end{aligned}
$$

Here we used a parabolic approximation for the term containing the Bessel functions. The prepore radius at the barrier, i.e., the critical radius, $r_{b}$, is determined by the maximum condition for $U(r, \sigma)$, which gives $r_{b}=\Gamma /(\sigma+B)$. Then the free energy of the barrier is $U_{b}=U\left(r_{b}\right)=\pi \Gamma^{2} /(\sigma+B)$. To explain the experimental results (i.e., that the rate constant for pore formation increases as the extent of electrostatic interaction increases), the condition $B>0$ must be satisfied to decrease $U_{\mathrm{b}}$. For the membrane systems $(X$ and $1 / \kappa)$ in this report, this condition corresponds to $a \leq 0.49$. Figure 4 shows examples of $U(r, \sigma)$ for different values of $C$ and $X$. Taking into account that the electrostatic interaction increases as $C$ decreases or $X$ increases, these graphs lead to the interpretation that the electrostatic interactions affect the free energy profile of the prepore, decreasing the $U_{\mathrm{b}}$ values.

We determined the rate constant for pore formation in charged membranes using the MFPT approach. According to the Kramers theory of Brownian motion [27, 28], evolution of a prepore is treated as a particle in stochastic motion in $r$-space within an asymmetric double-well potential $U(r)$. The walls with an infinite barrier at $r=0$ and a limited barrier at $r=r_{\mathrm{b}}$ work respectively as a reflecting boundary and an absorbing boundary. Then, the lifetime of the intact GUV, $\tau$, is defined as the time when the particle arrives at $r=r_{\mathrm{b}}$ and escapes from the hole, which is determined by the MFPT technique [27-29] as follows:

$$
\tau=\left(\frac{1}{D_{\mathrm{r}}}\right)_{0}^{r b} \exp [U(r) / k T]\left\{\int_{0}^{r} \exp [-U(z) / k T] d z\right\} d r
$$

where $D_{\mathrm{r}}$ is the diffusion coefficient of the particle in $r$-phase space. After some mathematical transformations [29], this expression can be written as follows:

$$
\tau=\left(\frac{k T}{D_{r}}\right)\left[\frac{2 \pi}{\sqrt{\partial^{2} U(0) / \partial r^{2}\left|\partial^{2} U\left(r_{b}\right) / \partial r^{2}\right|}}\right] \exp \left[\frac{U_{b}}{k T}\right]
$$


Applying this MFPT approach and the procedure commonly used for its simplification [28], we approximated $U(r)$ for $0<r<r_{b} / 2$ as $U(r)=3 \pi r^{2}(\sigma+B)$ and obtained the following equation from eq. (11b) (compare with eq. (7) in [6]):

$$
k_{\mathrm{p}}=k_{\mathrm{p}}(\sigma, X, \kappa)=\frac{1}{\tau}=\left(\frac{D_{r} \sqrt{3}}{k T}\right)(\sigma+B) \exp \left[-\frac{\pi \Gamma^{2}}{k T(\sigma+B)}\right] .
$$

\section{Discussion}

Based on the theory developed above and using eq. (12), we fit the experimental data in Fig. 2. This equation has three fitting parameters, $D_{\mathrm{r}}, a$, and $\Gamma$. The $D_{\mathrm{r}}$ values are determined mainly by an apparent frictional coefficient of the walk of the system in $r$-space. The latter is largely determined by rearrangement and conformational changes of lipids near the prepore and by the physical properties of the hydrocarbon chains. In this study, all of the hydrocarbon chains in the membranes were oleic acid. Therefore, we assumed here that the $D_{\mathrm{r}}$ values of all conditions are the same as $D_{\mathrm{r}}$ of a DOPC membrane $\left(=165 \mathrm{~nm}^{2} / \mathrm{s}\right)[6]$. For a specific value of $a$, the value of $\Gamma$ of the best-fit curve is determined. Figure 2 shows the best-fit curve for $a=$ 0.45. The data on the effect of salt concentration (Fig. 2a) at $C=312 \mathrm{mM}$ and $162 \mathrm{mM}$ fit the theoretical curves well, but at $C=12 \mathrm{mM}$, there was some deviation. The data on the effects of surface charge density (Fig. 2b) for $X=0.10$ and 0.40 fit the theoretical curves well, but for $X=0.70$, there was a large deviation. Deviations from theoretical results for highly charged GUVs and at low ionic strength can be explained reasonably well as a result of using a linear approximation of the Poisson-Boltzmann equation, which is valid if electric potential everywhere in system under consideration is less than $25 \mathrm{mV}$. The maximum value of the

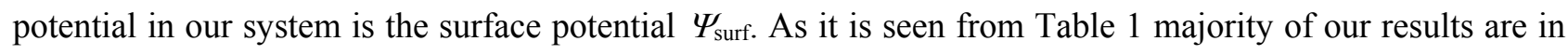
accord with this condition. But for $X=0.70$ and $C=162 \mathrm{mM} \Psi_{\text {surf }}=-60 \mathrm{mV}$ and for $X=0.40$ and $C=12$ $\mathrm{mM} \Psi_{\text {surf }}=-95 \mathrm{mV}$, and thereby the approximation of the Poisson-Boltzmann equation does not hold. To calculate the surface potential $\Psi_{\text {surf, }}$, we used the Graham equation [13] as follows: 


$$
\begin{aligned}
\psi_{\text {surf }} & =\frac{2 k T}{e} \sinh ^{-1}\left(\frac{\Omega_{\mathrm{p}}}{\sqrt{8 \varepsilon_{w} \varepsilon_{0} n R T}}\right) \\
& =51.4 \sinh ^{-1}\left(\frac{\Omega_{\mathrm{p}}}{0.117 \sqrt{C}}\right)[\mathrm{mV}] \text { at } 25^{\circ} \mathrm{C}
\end{aligned}
$$

In the case of $a=0.45$, the $\Gamma$ values were similar for different values of $C$ and $X$ (Table 1 ). For other values of $a$, we obtained similar results for the fit and $\Gamma$ values (Table 1). For lower values of $a(<0.40)$, the $\Gamma$ values increased a little with an increase in the extent of electrostatic interaction. At present, we don't have any experimental data on $\Gamma$ values of DOPG/DOPC membranes. Very recently, Pera et al. concluded that $\Gamma$ values for DOPG are smaller than for DOPC based on the self-consistent theory [30]. Therefore, the selection of the $a$ value ( $=0.45)$ is suitable based on the $\Gamma$ values. On the basis of the available experimental results, it is difficult to determine a unique combination of parameters $\left(D_{\mathrm{r}}, a\right.$, and $\left.\Gamma\right)$, but the results of the fit in Fig. 2 and the free energy profiles in Fig. 4 demonstrate that our hypothesis of how electrostatic interactions affect $k_{\mathrm{p}}$ reasonably explain the experimental results.

As we discussed above, the surface charge density of the prepore wall in our experiments was smaller than that of the bilayer surface (i.e., $a \leq 0.49$ ). This can be explained as follows. The cross-sectional area of the lipids in the wall of a toroidal prepore, $\beta A_{0}$ (where $\beta>1$ ), is larger than that in the interface of the planar bilayer, $A_{0}$, due to the increase in curvature of the monolayer forming the wall (Fig. 3a). If the DOPG molar fraction in the prepore wall is the same as that in the planar bilayer, the surface charge density on the prepore wall, $\Omega_{\mathrm{p}}$, equals $-e X / \beta A_{0}$. Thereby, $a=\Omega_{\mathrm{p}} / \Omega=1 / \beta=$ constant $(<1)$. This increase in the cross-sectional area of the lipids depends only on curvature of the rim of the prepore, and thereby the value of $\beta$ does not depend on prepore radius. This can reasonably explains the assumption that $\Omega_{\mathrm{p}}$ (i.e., $a$ ) does not depend on the prepore radius. To decide on an experimental value for $a$ among the many candidates in our analysis (Table 1), we need other experimental data such as the $\Gamma$ of a prepore in these membrane systems.

In this report, we used two approaches to consider the effects of the electrostatic interactions. To estimate the electrostatic interactions outside the bilayer, we used Lekkerkerker's approach, which is valid for any values of potential, i.e., which does not use the Debye-Hückel approximation. For electrostatic interactions inside the prepore we used a linearized Poisson-Boltzmann equation, i.e., the Debye-Hückel approximation, which is valid if the electric potential in the system is less than $25 \mathrm{mV}$. Moreover, the 
contribution of the electrostatic interaction inside the prepore to the rate constant of pore formation is small. This is supported by the fitting result; in Table 1, the case where $a=0$ means no surface charge density inside the prepore, and thereby, there is no contribution of the electrostatic interaction inside the prepore, but the fitting values of line tension are not greatly different from those in the charged prepore. This result clearly indicates that only the electrostatic interactions outside the bilayer plays a dominant role in the electrostatic effect on pore formation.

As far as we know, this report is the first one on quantitative effects of electrostatic interactions on mechanical stability of lipid membranes. Shoemaker et al. investigated the effect of the inclusion of anionic lipids on the critical tension inducing rupture of GUV using the elastic modulus measurement [31]. They found that critical tension decreased greatly with an increase in the concentration of anionic lipids in the membrane. This result suggests that the electrostatic interaction due to anionic lipids decreases the mechanical strength of the lipid membranes. Hence, this supports the results in this report. But they did not investigate the effect of salt concentration. Therefore it is difficult to conclude from this study whether the main factor affecting the critical tension is electrostatic interactions due to surface charges or changes in other physical properties due to the addition of other lipids. Moreover, values of the critical tension that induces rupture of GUVs are obtained during measurement of the elastic modulus, which depend on the experimental method; for example, decreasing the rate at which the applied tension is changed would greatly decrease the critical tension [3]. On the other hand, Evans et al. investigated the effect of hydrocarbon chain length on the rupture tension of a GUV using the dynamic tension spectroscopy and found that smaller tension induced rupture of lipid membranes with shorter chain length [2] and the rate constant of rupture of lipid membranes with shorter chain length is larger [3]. However they did not investigate the effect of surface charge densities on the rupture tension. As for planar lipid membranes, Meier et al. investigated rupture of black lipid membranes by short electric voltage pulse [32]. They found that the breakdown voltages of negatively charged phosphatidylserine (PS) membrane and electrically neutral phosphatidylcholine (PC) membrane were almost the same. Moreover, the ionic concentration did not greatly affect the breakdown voltage of the PS membrane. These results indicate that the electrostatic interactions do not play an important role in the breakdown of the planar lipid membranes. It is considered that tension in the membrane due to the applied voltage induces a pore and increases its size to the breakdown of the membrane [33]. At present, we don't know the reason of the discrepancy between our results using GUVs and their results using black lipid 
membranes.

The results in this report have an important biological implication. Pore formation in biological membranes can cause death of cells, and thus cells have had to modify their membrane structure (such as by incorporation of cholesterol into their plasma membranes [34] and addition of structural support by the cytoskeletons [35]) during their evolution. Moreover, there are many important physiological phenomena involved in pore formation in lipid membranes such as pore formation induced by antimicrobial peptides and pore-forming toxins [36,37]. The results in this report indicate that the electrostatic interactions due to surface charges must be taken into account for any realistic consideration of these phenomena.

\section{Conclusion}

In summary, we found that the rate constant $\left(k_{\mathrm{p}}\right)$ for pore formation induced under constant tension in lipid membranes increased with an increase in electrostatic interactions due to surface charges of the lipid membranes. This observation is very important because it not only offers a physical description of the phenomenon, but also its biological consequences. We developed a theory on the effect of the electrostatic interactions on the free energy profile of the membrane containing a prepore and also on the values of $k_{\mathrm{p}}$, which explains the experimental results and fits the experimental data reasonably well in the presence of weak electrostatic interactions. Based on these results, we conclude that a decrease in the free energy barrier of the prepore state due to electrostatic interactions is the main factor causing an increase in $k_{\mathrm{p}}$.

Acknowledgements: This work was supported in part by a Grant-in-Aid for Scientific Research (B) (No.15H04361) from Japan Society for the Promotion of Science (JSPS) to M.Y. Part of this research is based on a Cooperative Research Project of the Research Institute of Electronics, Shizuoka University.

\section{Appendix}

In this report we used the Poisson-Boltzmann equation to calculate electrostatic free energy due to the DEL (note that Lekkerkerker's equation (5) is derived from the Poisson-Boltzmann equation.). The general assumption when using the Poisson-Boltzmann equation is that the DEL is in an equilibrium state. In our system, a prepore opens and closes quickly (i.e., the radius of the prepore fluctuates). The question thus 
arises whether the Poisson-Boltzmann equation is applicable to such a system. The relaxation time of the DEL is determined by Debye length $\left(\lambda_{\mathrm{D}}\right)$ and the self-diffusion coefficient, $D_{\text {ion, }}$ of ions in aqueous solution, $t_{\mathrm{DEL}}=\left(\lambda_{\mathrm{D}}\right)^{2} / 2 D_{\text {ion }}$. The averaged self-diffusion coefficient of $\mathrm{Na}^{+}$and $\mathrm{Cl}^{-}$in water at room temperature is $D_{\mathrm{NaCl}}=1.6 \times 10^{-9} \mathrm{~m}^{2} / \mathrm{s} \quad[38]$. Hence, for $C=100 \mathrm{mM}\left(\lambda_{\mathrm{D}}=0.96 \mathrm{~nm}\right)$, the relaxation time of the DEL is equal to $t_{\mathrm{DEL}}=2.9 \times 10^{-10} \mathrm{~s}$. The characteristic time of evolution of the prepore, $t_{\text {pore }}=(r *)^{2} / 2 D_{\text {pore }}$, can be determined as the average time starting from $r=0$ to reach a prepore radius $r *$

For $D_{\text {pore }}=165 \mathrm{~nm}^{2} / \mathrm{s}$ (see Fig. 2), $t_{\text {pore }}$ for $r^{*}=1 \mathrm{~nm}$ is $6 \times 10^{-3} \mathrm{~s}$, which is $10^{7}$ times larger than the relaxation time of the DEL. Therefore, the DEL of the system under consideration can be treated as the equilibrium DEL during the total period of evolution of the prepore.

\section{References}

(1) O. Sandre, L. Moreaux, F. Brochard-Wyard, Proc. Natl. Acad. Sci. USA. 96, 10591 (1999).

(2) E. Evans, V. Heinrich, F. Ludwig, W. Rawicz, Biophys. J. 85, 2342 (2003)

(3) E. Evans, B. A. Smith, New J. Phys. 13, 095010 (2011).

(4) G. Fuerties, D. Giménez, S. Esteban-Martin, O. L. Sánchez-Muñoz, J. Salgado, Eur. Biophys. J. 40, 399 (2011).

(5) I. Gozen, P. Dommersnes, Eur. Phys. J. 223, 1813 (2014)

(6) V. Levadny, T. Tsuboi, M. Belaya, M.Yamazaki, Langmuir, 29, 3848 (2013).

(7) S. McLaughlin, D. Murray, Nature, 438, 605 (2005).

(8) M. Zasloff, Nature, 415, 389 (2002).

(9) Y. Tamba, M.Yamazaki, J. Phys. Chem. B, 113, 4846 (2009).

(10) Y. Tamba, H. Terashima, M. Yamazaki, Chem. Phys. Lipids, 164, 351 (2011)

(11) W. Rawicz, K. C. Olbrich, T. McIntosh, D. Needham, E. Evans, Biophys. J. 79, 328 (2000).

(12) It is considered that $1 \mathrm{mM}$ EGTA solution at $\mathrm{pH} 7.0$ contains $2 \mathrm{mM} \mathrm{Na}^{+}$based on the acid dissociation constant of EGTA.

(13) J. N. Israelachvili, Intermolecular and surface forces, $2^{\text {nd }}$ Ed. (Academic Press, New York, 1992). 
(14) J. Wohlert, W. K. den Otter, O. Edholm, W. J. Bries, J. Chem. Phys. 124, 154905 (2006).

(15) D. P. Tieleman, H. Leontiaudou, A. E. Mark, S.-J. Marrink, J. Amer. Chem. Soc. 125, 6382 (2003)

(16) E. Karatekin, O. Sandre, H. Guitouni, N. Borghi, P.-H. Puech, F. Brochart-Wyart, Biophys. J. 84, $1734(2003)$

(17) M. Fošnarič, V. Kralj-Iglič, B.K. Bohinc, S. May, J. Phys. Chem. B, 107, 12519 (2003).

(18) B. V. Deryagin, Y. V. Gutop, Kolloidn. Zh. (rus), 24, 370 (1962).

(19) J. D. Lister, Phys. Lett. A, 53, 193 (1975)

(20) M. D. Betterton, M. P. Brenner, Phys. Rev. Lett. 82, 1598 (1999).

(21) E. J. W. Verwey, J. Th. G. Overbeek, Theory of the stability of lyophobic colloids, (Elsevier, Amsterdam, 1948).

(22) R. J. Hunter, Foundation of Colloid Science, Vol. I, (Clarendon, Oxford Press, U. K., 1987).

(23) Under the tension, $A$ increases from $A_{0}$ by $\delta=\sigma / 4 \gamma$, where $\gamma$ is the interfacial energy per unit area at the bilayer-water interface [6]. The difference between $A$ and $A_{0}$ is less than $\sim 5 \%$, because $\sigma \leq 8$ $\mathrm{mN} / \mathrm{m}$ and $\gamma=39 \mathrm{mN} / \mathrm{m}$.

(24) J. F. Nagle, S. Tristram-Nagle, Biochim. Biophys. Acta 1469, 159 (2000)

(25) H. N. W. Lekkerkerker, Physica A, 159, 319 (1989)

(26) To circumvent the problem of divergence when $r \rightarrow 0$ we used here a slightly different definition of the DEL free energy, see [22].

(27) P. Hänggi, P. Talkner, M. Borkovec, Rev. Mod. Phys. 62, 251 (1990).

(28) C. W. Gardiner, Handbook of Stochastic Methods for Physics, Chemistry, and the Natural Sciences; 4th ed., (Springer-Verlag, Berlin, 2009).

(29) N. G. van Kampen, Stochastic processes in physics and chemistry, 3rd ed., (North Holland 2007)

(30) H. Pera, J. M. Kleijn, F. A. M. Leermakers, J. Chem. Phys. 142, 034101 (2015)

(31) S. D. Shoemaker, T. K. Vanderlick, Biophys. J. 83, 2007 (2002)

(32) W. Meier, A. Graff, A. Diederich, M. Winterhalter, Phys. Chem. Chem. Phys. 2, 4559 (2000)

(33) A. Diederich, M. Strobel, W. Meier, M. Winterhalter, J. Phys. Chem. B, 103, 1402 (1999)

(34) M. Bloom, O. G. Mouristen, In Structure and dynamics of membranes; R. Lipowsky, E Sackmann, eds., Elsevier Science B. V., Amsterdam, 1995; pp.65-95. 
(35) M. Yamazaki, S. Furuike, T. Ito, J. Muscle Research and Cell Motility, 23, 525 (2002)

(36) M. Z. Islam, J. M. Alam, Y. Tamba, M. A. S. Karal, M. Yamazaki, Phys. Chem. Chem. Phys., 16, 15752 (2014).

(37) M. A. S. Karal, J. M. Alam, T. Takahashi, V. Levadny, M. Yamazaki, Langmuir, 31, 3391 (2015).

(38) R. Milles, V. M. M. Lobe, Self Diffusion in Electrolyte Solutions, Elsevier, Amsterdam, 1989 


\section{Table 1}

(A) Effects of salt concentration

\begin{tabular}{|c|l|c|l|c|}
\hline \multirow{2}{*}{$a$} & \multicolumn{2}{|c|}{$C=312 \mathrm{mM}$} & \multicolumn{2}{c|}{$C=162 \mathrm{mM}$} \\
\cline { 2 - 5 } & $\begin{array}{c}\Gamma \\
(\mathrm{pN})\end{array}$ & $\begin{array}{c}\psi_{\text {surf }} \\
(\mathrm{mV})\end{array}$ & $\begin{array}{c}\Gamma \\
(\mathrm{pN})\end{array}$ & $\begin{array}{c}\psi_{\text {surf }} \\
(\mathrm{mV})\end{array}$ \\
\hline 0 & 13.9 & 0 & 14.3 & 0 \\
\hline 0.1 & 13.5 & -7 & 14.1 & -10 \\
\hline 0.2 & 13.0 & -14 & 13.8 & -19 \\
\hline 0.3 & 12.3 & -20 & 13.1 & -28 \\
\hline 0.4 & 11.3 & -27 & 12.1 & -36 \\
\hline 0.45 & 10.5 & -30 & 11.4 & -39 \\
\hline
\end{tabular}

(B) Effects of surface charge density

\begin{tabular}{|c|c|c|c|c|c|}
\hline \multirow{2}{*}{$a$} & $X=0$ & \multicolumn{2}{|c|}{$X=0.10$} & \multicolumn{2}{c|}{$X=0.40$} \\
\cline { 2 - 6 } & $\begin{array}{c}\Gamma \\
(\mathrm{pN})\end{array}$ & $\begin{array}{c}\Gamma \\
(\mathrm{pN})\end{array}$ & $\begin{array}{c}\psi_{\text {surf }} \\
(\mathrm{mV})\end{array}$ & $\begin{array}{c}\Gamma \\
(\mathrm{pN})\end{array}$ & $\begin{array}{c}\psi_{\text {surf }} \\
(\mathrm{mV})\end{array}$ \\
\hline 0 & 10.5 & 10.6 & 0 & 14.3 & 0 \\
\hline 0.1 & 10.5 & 10.6 & -2 & 14.1 & -10 \\
\hline 0.2 & 10.5 & 10.6 & -5 & 13.8 & -19 \\
\hline 0.3 & 10.5 & 10.5 & -7 & 13.1 & -28 \\
\hline 0.4 & 10.5 & 10.4 & -10 & 12.1 & -36 \\
\hline 0.45 & 10.5 & 10.4 & -11 & 11.4 & -39 \\
\hline
\end{tabular}

Table 1: The best-fit parameters for the data of $k_{\mathrm{p}}$ vs. tension shown in Fig. 2 and the surface potential. The best-fit parameters for the experimental data in Fig. 2 (i.e., tension dependence of $k_{\mathrm{p}}$ for DOPG/DOPC-GUVs for different salt concentrations and different values for fraction $X$ of DOPG) using $D_{\mathrm{r}}=165 \mathrm{~nm}^{2} / \mathrm{s}$. (a) Effect of salt concentration for constant $X(=0.40)$ (Fig. 2a). For each value of $a$, the best-fit curve was obtained with the $\Gamma$ value in the table. (b) Effect of surface charge density for constant $C$ (=162 mM) (Fig. 2b). For each value of $a$, the best-fit curve was obtained with the $\Gamma$ value in the table. 


\section{Figure legends}

Figure 1: (a) A differential interference contrast image of a GUV aspirated by a micropipet. $d$ is the internal diameter of the micropipet, and $D$ is the diameter of the spherical part of the GUV exterior to the micropipet. The bar indicates $10 \mu \mathrm{m}$. (b) Time course of the fraction of intact $40 \%$ DOPG/60\% DOPC-GUVs in a buffer $(C=12 \mathrm{mM})$ at two values for tension: $\sigma=3.0 \mathrm{mN} / \mathrm{m}(\circ)$ and $5.0 \mathrm{mN} / \mathrm{m}(\square)$. The solid line represents the best-fit curves by Eq. (2).

Figure 2: Tension dependence of $k_{\mathrm{p}}$ for DOPG/DOPC-GUVs for different values of fraction $X$ of DOPG and different salt concentrations $C$. Average values and standard errors of $k_{\mathrm{p}}$ for each tension were determined for three independent experiments using 20 GUVs for each experiment. The solid lines show the best-fit theoretical curves corresponding to eq. (12) with $D_{\mathrm{r}}=165 \mathrm{~nm}^{2} / \mathrm{s}$ and $a=0.45$. (a) Effect of salt concentration at constant $X(=0.40) . C=12 \mathrm{mM}(\circ), 162 \mathrm{mM}(\bullet)$, and $312 \mathrm{mM}(\square)$. The best-fit curves are shown for $C=$ $162 \mathrm{mM}(\Gamma=11.4 \mathrm{pN})$, and $312 \mathrm{mM}(\Gamma=10.5 \mathrm{pN})$. (b) Effect of surface charge density at constant $C(=162$ mM). 100\% DOPC (•), 10\% DOPG/90\% DOPC (口), 40\% DOPG/60\% DOPC (•), and 70\% DOPG/30\% DOPC (०). The best-fit curves are shown for $X=0(\Gamma=10.5 \mathrm{pN}), 0.10(\Gamma=10.4 \mathrm{pN})$, and $0.40(\Gamma=11.4$ $\mathrm{pN})$. The data for $100 \%$ DOPC GUVs and $40 \% \mathrm{DOPG} / 60 \%$ DOPC at $C=162 \mathrm{mM}$ are reprinted from Ref. [6] with permission from the American Chemical Society.

Figure 3: (a) A schematic drawing of a prepore in a lipid membrane which has a toroidal structure. (b) A cylinder model of the hydrophilic prepore with radius $r$ and height $h$.

Figure 4: Free energy profile $U(r)$ for different concentrations of solution $C$ and for different surface charge density values $X$ for constant tension $\sigma=7.0 \mathrm{mN} / \mathrm{m}$. (a) $X=0.40$ : (A) $C=450 \mathrm{mM}$; (B) $310 \mathrm{mM}$; (C) 160 mM; (D) $10 \mathrm{mM}$. (b) $C=160 \mathrm{mM}$; (A) $X=0$; (B) 0.20; (C) 0.40; (D) 0.70. In (a) and (b), U(r) was calculated according to eq. 9 using $\Gamma=10.5 \mathrm{pN}$ and $a=0.45$. 
Fig. 1

(a)

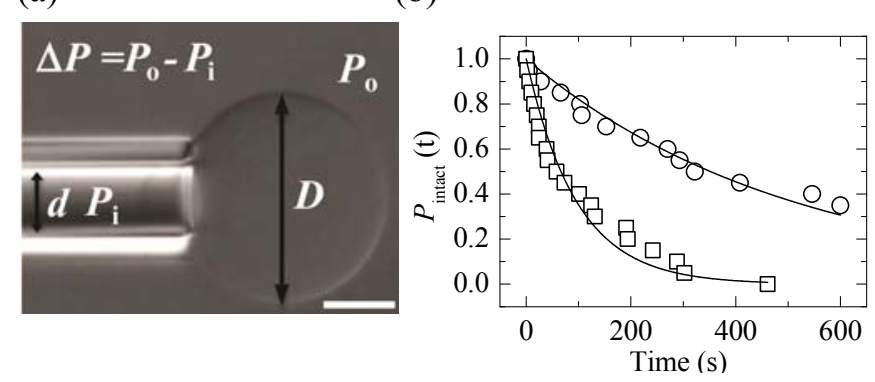


Fig. 2

(a)

(b)

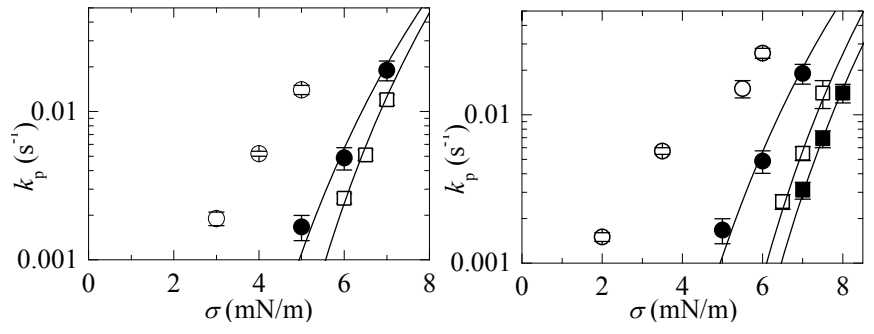


Fig. 3.

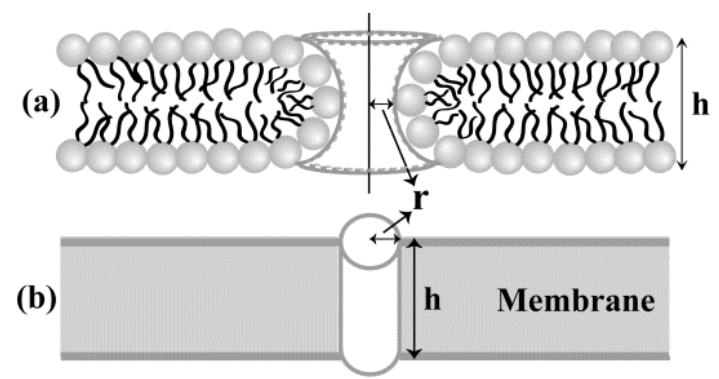


Fig. 4.

(a)

(b)
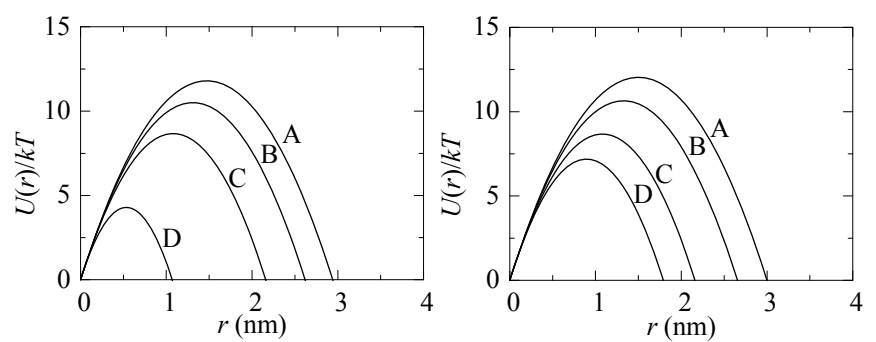